\title{
LINIERITAS INTEGRAL HENSTOCK-PETTIS PADA RUANG EUCLIDE Rn
}

\author{
Hairur Rahman \\ Jurusan Matematika Fakultas Sains dan Teknologi \\ Universitas Islam Negeri Maulana Malik Ibrahim Malang
}

\begin{abstract}
In this paper we study Henstock-Pettis integral on the Euclidean space $\mathfrak{R}^{n}$. We discuss some properties Linear integrable.
\end{abstract}

Keywords: Henstock integral, Euclidean Space $\Re^{n}$, properties Linear integrable, Henstock-Pettis integral.

\section{PENDAHULUAN}

Pada tahun 1914, Perron mengembangkan perluasan lain integral Lebesgue dan menunjukkan bahwa integralnya mempunyai sifat bahwa setiap derivatifnya terintegral pada garis lurus. Selanjutnya Henstock dan Kurzweil secara terpisah mengitlakkan integral Riemann dengan mengubah konstanta positif $\delta$ menjadi fungsi positif $\delta$ dan ternyata integral yang disusun keduanya ekuivalen. Oleh karena itu, integral yang mereka susun terkenal dengan nama Henstock-Kurzweil (Lee dan Vborn, 2000).

Dari kajian tentang integral Henstock banyak sifat-sifat yang telah diungkapkan baik dalam $\mathfrak{R}$ maupun ruang $\mathfrak{R}^{n}$. Menurut penelitian, masalah mengenai sifat-sifat pada integral Henstock kemungkinan dapat dikembangkan menjadi masalah yang lebih luas dalam integral Henstock-Pettis, khususnya sejauh mana sifatsifat integral Henstock dari fungsi bernilai real dapat dikembangkan ke dalam integral HenstockPettis pada ruang Euclide $\mathfrak{R}^{n}$. Dari kajian tentang integral Henstock-Kurzweil banyak sifat-sifat yang telah diungkapkan baik dalam $\mathfrak{R}$ maupun ruang $\mathfrak{R}^{n}$. Menurut penelitian sebelumnya, masalah mengenai sifat-sifat pada integral Henstock-Kurzweil yang telah dilakukan oleh Guoju dan Tianqing, 2001, Guoju dan Swabik, 2001, Indrati, 2002, serta Dharmawidjaya, 2003, kemungkinan dapat dikembangkan menjadi masalah yang lebih luas dalam integral HenstockKurzweil-Pettis, khususnya sejauh mana sifatsifat integral Henstock dari fungsi bernilai real dapat dikembangkan ke dalam integral HenstockKurzweil-Pettis pada ruang Euclide $\mathfrak{R}^{n}$.

Definisi 1.1. Diberikan fungsi volume $\alpha$ pada $\mathfrak{R}^{n}$, sel $A \subset \mathfrak{R}^{n}$ dan $E$ ruang Banach. Fungsi $f: E \rightarrow X$ dikatakan terintegral- $\alpha$ Henstock pada E terhadap $\alpha$, ditulis singkat $f \in \mathcal{H}(E, \alpha, X)$ jika terdapat vektor $A \in X$ sehingga untuk setiap bilangan $\varepsilon>0$ terdapat fungsi positif $\delta$ pada $E$ dan untuk setiap partisi Perron $\delta$-fine

$\mathcal{D}=\left\{\left(D_{1}, \bar{x}_{1}\right),\left(D_{2}, \bar{x}_{2}\right), \cdots,\left(D_{n}, \bar{x}_{n}\right)\right\}$ pada E berlaku

$$
\begin{aligned}
\left\|A-(\mathcal{D}) \sum f(x) \alpha(D)\right\| \\
=\left\|A-(\mathcal{D}) \sum_{i=1}^{n} f\left(\bar{x}_{i}\right) \alpha\left(D_{i}\right)\right\|<\varepsilon .
\end{aligned}
$$

\section{PEMBAHASAN}

Akan dibahas sifat-sifat lanjut dari integral Henstock-Pettis pada ruang Euclide $\mathfrak{R}^{n}$, yakni memuat pembahasan yang berkaitan dengan sifat-sifat Linearitas fungsi terintegral HenstockPettis pada ruang Euclide $\mathfrak{R}^{n}$.

Definisi 2.1. Diberikan $X$ ruang Banach dan $X^{*}$ ruang dualnya, volume $\alpha$ pada $\mathfrak{R}^{n}$, dan se $A \subset \mathfrak{R}^{n}$. Fungsi $f: E \rightarrow X$ dikatakan terintegral- $\alpha$ HenstockPettis pada $E$, ditulis singkat dengan $f \in \mathcal{H} \mathcal{P}(E, \alpha)$, jika untuk setiap $x^{*} \in X^{*}$ dan sel $A \subset E$, fungsi $x^{*} f$ terintegral- $\alpha$ Henstock pada $A$ dan terdapat vektor $x_{(f, A, \alpha)} \in X$ sehingga

$$
x^{*}\left(x_{(f, A, \alpha)}\right)=(\mathcal{H}) \int_{A} x^{*} f d \alpha .
$$

Selanjutnya vektor $x_{(f, A, \alpha)}$ di atas disebut nilai Integral Henstock-Pettis fungsi f pada A dan ditulis

khususnya

$$
x_{(f, A, \alpha)}=(\mathcal{H} \mathcal{P}) \int_{A} f d \alpha,
$$

Jadi

$$
x_{(f, E, \alpha)}=(\mathcal{H} \mathcal{P}) \int_{E} f d \alpha
$$

$$
x^{*}\left((\mathcal{H} \mathcal{P}) \int_{A} f d \alpha,\right)=(\mathcal{H}) \int_{A} x^{*} f d \alpha .
$$

Teorema 2.2. Jika $f \in \operatorname{HP}(E, \alpha)$, maka vektor $x_{(f, A, \alpha)} \in X$ yang dimaksud di dalam Definisi 2.1 adalah tunggal

Bukti: Jika terdapat vektor $x_{1(f, A, \alpha)}$ dan $x_{2}(f, A, \alpha)$ seperti pada definisi 2.1 maka

$$
x^{*}\left(x_{1(f, A, \alpha)}\right)=(\mathcal{H}) \int_{A} x^{*} f d \alpha
$$


dan

$$
x^{*}\left(x_{2_{(f, A, \alpha)}}\right)=(\mathcal{H}) \int_{A} x^{*} f d \alpha
$$

oleh karena itu,

$$
\begin{aligned}
x^{*}\left(x_{1_{(f, A, \alpha)}}\right)- & x^{*}\left(x_{2(f, A, \alpha)}\right) \\
& =(\mathcal{H}) \int_{A} x^{*} f d \alpha-(\mathcal{H}) \int_{A} x^{*} f d \alpha
\end{aligned}
$$

atau

$$
x^{*}\left(x_{1_{(f, A, \alpha)}}\right)=x^{*}\left(x_{2_{(f, A, \alpha)}}\right)
$$

Untuk setiap $x^{*} \in X^{*}$

Jadi

$$
x_{1_{(f, A, \alpha)}}=x_{2_{(f, A, \alpha)}}
$$

Contoh 2.3 Jika $x^{*} f(\bar{x})=c$ untuk setiap $\bar{x}$ di dalam sel $E \subset \mathfrak{R}^{n}$, maka $f \in H P(E, \alpha)$ dan

$$
x^{*}\left(x_{(f, A, \alpha)}\right)=\alpha(A) c .
$$

Bukti : Diberikan sebarang bilangan $\varepsilon>0$ dan $x^{*} \in X^{*}$ maka dapat ditemukan fungsi positif $\delta$ pada $E$ sehingga jika $A \subset E$ sebarang sel dan $\mathcal{D}=\{(D, \bar{x})\}$ sebarang partisi Perron $\delta$-fine pada sel A, maka

$$
\begin{aligned}
\left|(\mathcal{D}) \sum x^{*} f(\bar{x}) \alpha(D)-x^{*}\left(x_{(f, A, \alpha)}\right)\right| \\
=\left|(\mathcal{D}) \sum c \alpha(D)-\alpha(A) c\right| \\
=|\alpha(A) c-\alpha(A) c|=0<\varepsilon
\end{aligned}
$$

Jadi $x^{*} f$ terintegral Henstock pada $E$ dan untuk sel $A \subset E$ di atas, maka terdapat vektor $x_{(f, A, \alpha)} \in X$ sehingga

$$
\begin{aligned}
x^{*}\left(x_{(f, A, \alpha)}\right) & =(\mathcal{H}) \int_{A} x^{*} f d \alpha \\
& =(\mathcal{H}) \int_{A} c d \alpha \\
& =\alpha(A) c
\end{aligned}
$$

Jadi $f$ terintegral Henstock-Pettis pada $E$.

Teorema 2.4 Diberikan $X$ ruang Banach dan $X^{*}$ dualnya, volume $\alpha$ pada $\Re^{n}$ dan sel $E \subset \Re^{n}$.

(i) Jika f,g $\in \mathcal{H} \mathcal{P}(E, \alpha)$ maka $f+g \in \mathcal{H} \mathcal{P}(E, \alpha)$

$$
\operatorname{dan} x_{(f+g, A, \alpha)}=x_{(f, A, \alpha)}+x_{(g, A, \alpha)}
$$

(ii) Jika $f \in \mathcal{H} \mathcal{P}(E, \alpha)$ dan $c \in \Re$ maka

$c f \in \mathcal{H} \mathcal{P}(E, \alpha)$ dan $x_{(c f, A, \alpha)}=c . x_{(f, A, \alpha)}$

\section{Bukti :}

(i). Fungsi $f \in \mathcal{H} \mathcal{P}(E, \alpha)$, maka untuk setiap $x^{*} \in X^{*}$ fungsi $x^{*} f$ terintegral Henstock pada $E$ dan untuk setiap sel $A \subset E$ terdapat vektor $x_{(f, A, \alpha)} \in X$ sehingga

$$
x^{*}\left(x_{(f, A, \alpha)}\right)=(\mathcal{H}) \int_{A} x^{*} f d \alpha
$$

dan fungsi $g \in \mathcal{H} \mathcal{P}(E, \alpha)$, maka untuk setiap $x^{*} \in X^{*}$ fungsi $x^{*} g$ terintegral Henstock pada $E$ dan untuk setiap sel $A \subset E$ terdapat vektor $x_{(g, A, \alpha)} \in X$ sehingga

$$
x^{*}\left(x_{(g, A, \alpha)}\right)=(\mathcal{H}) \int_{A} x^{*} g d \alpha
$$

Karena $A \subset E$ sebarang sel, maka berlaku untuk fungsi $f$ dan fungsi $g$. Jadi untuk setiap $x^{*} \in X^{*}$ fungsi $x^{*}(f+g)$ terintegral Henstock pada $E$. Oleh karena itu terdapat vektor $x_{(f+g, A, \alpha)} \in X$ sehingga untuk sel $A \subset E$ di atas berlaku

$$
\begin{aligned}
x^{*}\left(x_{(f+g, A, \alpha)}\right)=(\mathcal{H}) \int_{A} x^{*}(f+g) d \alpha \\
=(\mathcal{H}) \int_{A} x^{*} f d \alpha+(\mathcal{H}) \int_{A} x^{*} g d \alpha \\
=x^{*}\left(x_{(f, A, \alpha)}\right)+x^{*}\left(x_{(g, A, \alpha)}\right)
\end{aligned}
$$

Jadi $f+g \in \mathcal{H} \mathcal{P}(E, \alpha)$

dan

$$
x_{(f+g, A, \alpha)}=x_{(f, A, \alpha)}+x_{(g, A, \alpha)}
$$

(ii). Fungsi $f \in \mathcal{H} \mathcal{P}(E, \alpha)$, maka untuk setiap $x^{*} \in X^{*}$ fungsi $x^{*} f$ terintegral Henstock pada $E$ dan untuk setiap sel $A \subset E$ terdapat vektor $x_{(f, A, \alpha)} \in X$ sehingga

$$
x^{*}\left(x_{(f, A, \alpha)}\right)=(\mathcal{H}) \int_{A} x^{*} f d \alpha
$$

Oleh karena itu, untuk setiap $x^{*} \in X^{*}$ dan $c \in \Re$ maka $x^{*} c f$ terintegral Henstock pada $E$ dan untuk setiap sel $A \subset E$ terdapat vektor $x_{(c f, A, \alpha)} \in X$.

Sehingga

Jadi $c f \in \mathcal{H} \mathcal{P}(E, \alpha)$

$$
\begin{aligned}
x^{*}\left(x_{(c f, A, \alpha)}\right) & =(\mathcal{H}) \int_{A} x^{*} c f d \alpha \\
& =c(\mathcal{H}) \int_{A} x^{*} f d \alpha \\
& =c x^{*}\left(x_{(f, A, \alpha)}\right)
\end{aligned}
$$

dan

$$
x_{(c f, A, \alpha)}=c \cdot x_{(f, A, \alpha)}
$$

Teorema 2.5 Diberikan $X$ ruang Banach dan $X^{*}$ dualnya, volume $\alpha$ dan $\beta$ di dalamnya $\Re^{n}$ dan sel $E \subset \mathfrak{R}^{n}$.

(i). Jika $f \in \mathcal{H} \mathcal{P}(E, \alpha)$ dan $f \in \mathcal{H} \mathcal{P}(E, \beta)$, maka $f \in$ $\mathcal{H P}(E, \alpha+\beta)$

$$
\operatorname{dan} x_{(f, A, \alpha+\beta)}=x_{(f, A, \alpha)}+x_{(f, A, \beta)}
$$

(ii). Jika $f \in \operatorname{HP}(E, \alpha)$ dan $e \in \mathfrak{R}$ dengan e $>0$ maka $f \in \operatorname{HP}(E, e \alpha) \operatorname{dan} x_{(f, A, e \alpha)}=e \cdot x_{(f, A, \alpha)}$

\section{Bukti :}

(i). Fungsi $f \in \mathcal{H} \mathcal{P}(E, \alpha)$, maka untuk setiap $x^{*} \in X^{*}$ fungsi $x^{*} f$ terintegral Henstock pada $E$ dan untuk setiap sel $A \subset E$ terdapat vektor $x_{(f, A, \alpha)} \in X$ sehingga 


$$
x^{*}\left(x_{(f, A, \alpha)}\right)=(\mathcal{H}) \int_{A} x^{*} f d \alpha
$$

dan fungsi $f \in \mathcal{H} \mathcal{P}(E, \beta)$, maka untuk setiap $x^{*} \in X^{*}$ fungsi $x^{*} f$ terintegral Henstock pada $E$ dan untuk setiap sel $A \subset E$ terdapat vektor $x_{(f, A, \beta)} \in X$ sehingga

$$
x^{*}\left(x_{(f, A, \beta)}\right)=(\mathcal{H}) \int_{A} x^{*} f d \beta
$$

Karena $A \subset E$ sebarang sel, maka berlaku untuk fungsi $f$ yang terintegral HenstockPettis pada $E$ terhadap $\alpha$ dan $\beta$.

Jadi untuk setiap $x^{*} \in X^{*}$ fungsi $x^{*} f$ terintegral Henstock pada $E$.

Oleh karena itu terdapat vektor $x_{(f, A, \alpha+\beta)} \in X$ sehingga sel $A \subset E$ di atas berlaku

$$
\begin{aligned}
x_{(f, A, \alpha+\beta)} & =(\mathcal{H}) \int_{A} x^{*} f d(\alpha+\beta) \\
& =(\mathcal{H}) \int_{A} x^{*} f d \alpha+(\mathcal{H}) \int_{A} x^{*} f d \beta \\
& =x_{(f, A, \alpha)}+x_{(f, A, \beta)}
\end{aligned}
$$

Jadi $f \in \mathcal{H} \mathcal{P}(E, \alpha+\beta)$,

dan

$$
x_{(f, A, \alpha+\beta)}=x_{(f, A, \alpha)}+x_{(f, A, \beta)}
$$

(ii). Fungsi $f \in \mathcal{H} \mathcal{P}(E, \alpha)$, maka untuk setiap $x^{*} \in X^{*}$ fungsi $x^{*} f$ terintegral Henstock pada $E$ dan untuk setiap sel $A \subset E$ terdapat vektor $x_{(f, A, \alpha)} \in X$ sehingga

$$
x^{*}\left(x_{(f, A, \alpha)}\right)=(\mathcal{H}) \int_{A} x^{*} f d \alpha
$$

Oleh karena itu, jika $e \in \mathfrak{R}$ dengan $e>0$ maka untuk sel $A \subset E$ di atas terdapat vektor $x_{(f, A, e \alpha)} \in X$ sehingga

$$
\begin{aligned}
x^{*}\left(x_{(f, A, e \alpha)}\right) & =(\mathcal{H}) \int_{A} x^{*} f d e \alpha \\
& =e(\mathcal{H}) \int_{A} x^{*} f d \alpha \\
& =e x^{*}\left(x_{(f, A, \alpha)}\right)
\end{aligned}
$$

Jadi $f \in \mathcal{H} \mathcal{P}(E$, e $\alpha)$

dan

$$
x_{(f, A, e \alpha)}=e \cdot x_{(f, A, \alpha)}
$$

Teorema 2.6 Jika $E_{1}, E_{2} \subset \Re^{n}$ sel-sel tak saling tumpang tindih, $f \in \mathcal{H} \mathcal{P}\left(E_{1}, \alpha\right)$ dan, $f \in \mathcal{H} \mathcal{P}\left(E_{2}, \alpha\right)$ maka, $f \in \mathcal{H} \mathcal{P}\left(E_{1} \cup E_{2}, \alpha\right)$ dan jika $A \subset E_{1}$ sel dan $B \subset E_{2}$ sel maka

$$
x_{(f, A \cup B, \alpha)}=x_{(f, A, \alpha)}+x_{(f, B, \alpha)}
$$

Bukti : Diberikan sebarang bilangan $\varepsilon>0$ dan $x^{*} \in X^{*}$, maka terdapat fungsi positif $\delta_{1}$ pada $E_{1}$ dan $\delta_{2}$ pada $E_{2}$ sehingga untuk $A \subset E_{1}$ sebarang sel dan $B \subset E_{2}$ sebarang sel dan $\mathcal{D}_{1}=\{(D, \bar{x})\}$ dan $D_{2}=\{(D, \bar{x})\}$ berturut-turut partisi Perron $\delta$-fine pada $A$ dan $B$ berlaku

$$
\left|\left(\mathcal{D}_{1}\right) \sum x^{*} f(\bar{x}) \alpha(D)-x^{*}\left(x_{(f, A, \alpha)}\right)\right|<\frac{\varepsilon}{2}
$$

dan

$$
\begin{array}{rl}
\mid\left(\mathcal{D}_{2}\right) \sum x^{*} & f(\bar{x}) \alpha(D)-x^{*}\left(x_{(f, A, \alpha)}\right) \mid<\frac{\varepsilon}{2} \\
& =\left|(\mathcal{D}) \sum c \alpha(D)-\alpha(A) c\right| \\
& =|\alpha(A) c-\alpha(A) c|=0<\varepsilon
\end{array}
$$

Diambil fungsi $\delta$ :

$$
\delta(\bar{x})=\left\{\begin{array}{cc}
\delta_{1}(\bar{x}) & , \text { jika } \bar{x} \in A \text { dan } \bar{x} \notin B \\
\delta_{2}(\bar{x}) & , \text { jika } \bar{x} \in B \text { dan } \bar{x} \notin A \\
\min \left(\delta_{1}(\bar{x}),\right. & \left., \delta_{2}(\bar{x})\right), \text { jika } \bar{x} \in A \cap B
\end{array}\right.
$$

Diperoleh $\delta$ fungsi positif pada $E_{1} \cup E_{2}$ dan untuk sel-sel $A \subset E_{1}$, dan $B \subset E_{2}$ di atas dan $\mathrm{D}_{1} \cup \mathrm{D}_{2}$ partisi Perron $\delta$ fine pada $A \cup B$. Oleh karena itu diperoleh

$$
\begin{aligned}
\mathrm{I}\left(\mathcal{D}_{1} \cup \mathcal{D}_{2}\right) \sum x^{*} f(\bar{x}) \alpha(D)- & \\
& \left(x^{*}\left(x_{(f, A, \alpha)}\right)+x^{*}\left(x_{(f, A, \beta)}\right)\right) \mid \\
= & \mid\left(\left(\mathcal{D}_{1}\right) \sum x^{*} f(\bar{x}) \alpha(D)+\left(\mathcal{D}_{2}\right) \sum x^{*} f(\bar{x}) \alpha(D)\right)- \\
& \left(x^{*}\left(x_{(f, A, \alpha)}\right)+x^{*}\left(x_{(f, A, \beta)}\right)\right) \mid \\
= & \mid\left(\left(\mathcal{D}_{1}\right) \sum x^{*} f(\bar{x}) \alpha(D)+\left(\mathcal{D}_{2}\right) \sum x^{*} f(\bar{x}) \alpha(D)\right)- \\
& \left(x^{*}\left(x_{(f, A, \alpha)}\right)+x^{*}\left(x_{(f, A, \beta)}\right)\right) \mid \\
\leq & \mid\left(\left(\mathcal{D}_{1}\right) \sum x^{*} f(\bar{x}) \alpha(D)+x^{*}\left(x_{(f, A, \alpha)}\right) \mid+\right. \\
& \mid\left(\left(\mathcal{D}_{2}\right) \sum x^{*} f(\bar{x}) \alpha(D)+x^{*}\left(x_{(f, A, \beta)}\right) \mid\right. \\
< & \frac{\varepsilon}{2}+\frac{\varepsilon}{2}=\varepsilon
\end{aligned}
$$

Jadi $x^{*} f$ terintegral Henstock pada $E_{1} \cup E_{2}$ dan untuk sel-sel $A \subset E_{1}, B \subset E_{2}$ di atas terdapat vektor $\left(x_{(f, A \cup B, \alpha)}\right) \in X$ sehingga

$$
\begin{aligned}
x^{*}\left(x_{(f, A \cup B, \alpha)}\right) & =(\mathcal{H}) \int_{A \cup B} x^{*} f d \alpha \\
& =(\mathcal{H}) \int_{A} x^{*} f d \alpha+(\mathcal{H}) \int_{B} x^{*} f d \alpha \\
& =x^{*}\left(x_{(f, A, \alpha)}\right)+x^{*}\left(x_{(f, B, \alpha)}\right)
\end{aligned}
$$

Jadi $f \in \mathcal{H} \mathcal{P}\left(E_{1} \cup E_{2}, \alpha\right)$

dan

$$
x_{(f, A \cup B, \alpha)}=x_{(f, A, \alpha)}+x_{(f, B, \alpha)}
$$

Selanjutnya teorema di bawah ini merupakan akibat teorema 2.6.

Teorema 2.7 Diberikan sel $E \subset \mathfrak{R}^{n}$. Jika $A \subset E$ sel dan $\mathrm{D}=\left\{\left(D_{1}, D_{2}, \ldots, D_{m}\right)\right\}$ divisi pada $A$ serta $f \in \mathcal{H} \mathcal{P}\left(D_{i}, \alpha\right)$ untuk setiap i, maka $f \in \mathcal{H} \mathcal{P}(E, \alpha)$ dan

$$
x_{(f, A, \alpha)}=\sum_{i=1}^{m} x_{\left(f, D_{i}, \alpha\right)}
$$

Bukti : Diberikan sebarang bilangan $\varepsilon>0$ dan $x^{*} \in X^{*}$, maka terdapat fungsi positif $\delta$ dan $D_{i}$, sehingga jika $\left\{\left(B_{k}, \bar{x}_{k}\right): k=1,2, \ldots, p\right\}$ partisi Perron $\delta_{i}$-fine pada $D_{i}$ berlaku 


$$
\left|x^{*}\left(x_{\left(f, D_{i}, \alpha\right)}\right)-\sum_{k=1}^{p} x^{*} f\left(\bar{x}_{k}\right) \alpha\left(B_{k}\right)\right|<\frac{\varepsilon}{m}
$$

untuk setiap $i=1,2, . ., m$.

Dibentuk fungsi positif $\delta$ :

$$
\delta(\bar{x})= \begin{cases}\delta_{i}(\bar{x}) & , \bar{x} \in \operatorname{int}\left(D_{i}\right), i=1,2, \ldots, m \\ \min \left\{\delta_{i}(\bar{x})\right\} & , \bar{x} \in \partial\left(D_{i}\right) \text { utk bbrp } \bar{x} \in D_{i}\end{cases}
$$

diperoleh $\delta$ fungsi positif pada $E$ dan $\delta(\bar{x}) \leq \delta_{i}(\bar{x})$ untuk setiap $\bar{x} \in D_{i}, i=1,2, \ldots, m$.

Diambil sebarang partisi Perron $\delta$-fine $\mathrm{P}_{i}$ pada $D_{i}$ untuk setiap $i=1,2, \ldots, m$.

Menurut Teorema 2.6.2., $P=\bigcup_{i=1}^{m} P_{i}$ merupakan partisi Perron $\delta$-fine pada $E$.

Akibatnya, untuk $A \subset E$ sebarang sel $\mathrm{P}$ merupakan partisi Perron $\delta$-fine pada $A$. Oleh karena itu $\mathrm{P}_{i}$ merupakan partisi Perron $\delta$-fine $D_{i}$ untuk setiap $i=1,2, \ldots, m$, maka diperoleh

$$
\begin{aligned}
& \left|\sum_{i=1}^{m} x^{*}\left(x_{\left(f, D_{i}, \alpha\right)}\right)-\mathcal{P} \sum_{i=1}^{m} x^{*} f(\bar{y}) \alpha(P)\right| \\
& \leq\left|\sum_{i=1}^{m} x^{*}\left(x_{\left(f, D_{i}, \alpha\right)}\right)-\sum_{i=1}^{m} \mathcal{P}_{i} x^{*} f(\bar{y}) \alpha(P)\right| \\
& \leq\left|\sum_{i=1}^{m} x^{*}\left(x_{\left(\left(f, D_{1}, \alpha\right)\right)}\right)-\sum_{i=1}^{m} \boldsymbol{P}_{i} \sum x^{*} f(\bar{y}) \alpha(P)\right| \\
& \leq\left|\sum_{i=1}^{m} x^{*}\left(x_{\left(\left(f, D_{1}, \alpha\right)\right)}\right)-\boldsymbol{P}_{i} \sum x^{*} f(\bar{y}) \alpha(P)\right| \\
& <\sum_{i=1}^{m} \frac{\mathcal{E}}{m}=\varepsilon
\end{aligned}
$$

Jadi untuk setiap $x^{*} \in X^{*}$ fungsi $x^{*} f$ terintegral Henstock pada $E$ dan untuk setiap sel $A \subset E$ di atas terdapat vektor $x_{(f, A, \alpha)} \in X$ sehingga

$$
\begin{aligned}
x^{*}\left(x_{(f, A, \alpha)}\right) & =\sum_{i=1}^{m}(\mathrm{H}) \int_{D_{i}} x^{*} f d \alpha \\
& =\sum_{i=1}^{m} x^{*}\left(x_{\left(f, D_{i}, \alpha\right)}\right)
\end{aligned}
$$

Jadi $f \in \mathrm{HP}(\mathrm{E}, \alpha)$

dan

$$
x^{*}\left(x_{(f, A, \alpha)}\right)=\sum_{i=1}^{m} x_{\left(f, D_{i}, \alpha\right)}
$$

Teorema 2.8. Jika $f=0$ (fungsi nol) $h, d$ pada $E$ maka $f \in \operatorname{HP}(E, \alpha)$ dan jika $A \subset E$ sel maka

$$
x_{(f, A, \alpha)}=\theta
$$

Bukti : Karena $f=0$ h.d pada $E$, maka terdapat himpunan $A \subset E$ dengan $\mu_{\alpha}(A)=0$ sehingga jika $x^{*} \in X^{*}$ berakibat

$$
x^{*} f(\bar{x})\left\{\begin{array}{l}
=0, \text { jika } \bar{x} \in E-A \\
\neq 0, \text { jika } x \in A
\end{array}\right.
$$

Dibentuk $A=\bigcup_{i=1}^{\infty} A_{i} \operatorname{dimana}$

$$
A_{i}=\{\bar{x} \in A: i-1 \leq\|f(\bar{x})\| \leq i, i=1,2, \ldots . .\} A
$$

dengan $\mu_{\alpha}\left(A_{i}\right)=0$

Diberikan bilangan $\varepsilon>0$. Untuk setiap $i$ terdapat himpunan terbuka $G_{i}$ dengan ukuran kurang dari

$$
\frac{\varepsilon}{i 2^{i}} \text { sehingga } G_{i} \supset A_{i} \text {. }
$$

Didefinisikan fungsi positif $\delta$ pada $E$ sedemikian sehingga $N(\bar{x}, \delta(\bar{x})) \subset G_{i}$, untuk $\bar{x} \in A_{\mathrm{i}}, i=1,2, \ldots$, dan sebarang fungsi positif untuk $\bar{x}$ yang lain . Oleh karena itu untuk sebarang $A \subset E$ sel dan sebarang partisi Perron $\delta$-fine $\mathrm{P}=\{(B, \bar{x})\}$ pada $A$ berlaku

$$
\begin{aligned}
& \left|(\boldsymbol{D}) \sum x^{*} f(x) \alpha(A)-0\right| \\
& =\left|\sum_{x \in A_{i}} x^{*} f(x) \alpha(A)+\sum_{x \notin A_{i}} x^{*} f(x) \alpha(A)\right| \\
& =\left|\sum_{x \in A_{i}} x^{*} f(x) \alpha(A)\right| \\
& <\sum_{i=1}^{\infty}\left\|x^{*}\right\| i \frac{\mathcal{E}}{2^{i} \cdot i} \\
& =\varepsilon
\end{aligned}
$$

untuk setiap $x^{*} \in X^{*}$ dan $\left\|x^{*}\right\| \leq 1$.

Hal ini menunjukkan bahwa untuk setiap $x^{*} \in X^{*}$ fungsi $x^{*} f$ terintegral Henstock pada E.

dan untuk sel $A \subset E$ di atas terdapat vektor $x_{(f, A, \alpha)} \in X$ sehingga

$$
\begin{aligned}
x^{*}\left(x_{(f, A, \alpha)}\right) & =(\not) \int_{A} x^{*} f d \alpha \\
& =(\not) \int 0 d \alpha=\alpha(A) 0=0
\end{aligned}
$$

Jadi $f \in \operatorname{HP}(E, \alpha)$

dan

$$
x_{(f, A, \alpha)}=\theta
$$

Berikut ini akibat Teorema 3.1.8 di atas.

Teorema 3.1.9. Jika $f \in \operatorname{HP}(E, \alpha)$ dan $g=f$ h.d pada $E$ maka $g \in \mathrm{HP}(E, \alpha)$ dan jika $A \subset$ E sel maka

$$
x_{(g, A, \alpha)}=x_{(f, A, \alpha)}
$$


Bukti : ambil fungsi $h=g-f$. Diperoleh $h=0$ (fungsi nol) h.d pada $E$. Menurut Teorema 3.1.8, $h$ $\in \operatorname{HP}(E, \alpha)$ dan

untuk setiap sel $A \subset E$.

$$
x_{(h, A, \alpha)}=\theta
$$

Karena $h \in \operatorname{HP}(E, \alpha), f \in \operatorname{HP}(E, \alpha)$ dan $g=h+f$, maka berdasarkan Teorema

diperoleh, $g \in \mathrm{HP} \quad(E, \alpha)$ dan untuk setiap $x^{*} \in X^{*}$

$$
x^{*}\left(x_{(g, A, \alpha)}\right)=(\not) \int_{A} x^{*}(h+f) d \alpha
$$$$
=(\not{Z}) \int_{A} x^{*} h d \alpha+(\not{Z}) \int_{A} x^{*} f d \alpha
$$$$
=0+(\not) \int_{A} x^{*} f d \alpha
$$$$
=(\not) \int_{A} x^{*} f d \alpha
$$$$
=x^{*}\left(\begin{array}{l}
A \\
x_{(f, A, \alpha)}
\end{array}\right)
$$

Jadi

$$
x_{(g, A, \alpha)}=x_{(f, A, \alpha)}
$$

Teorema 3.1.10. Jika $f \in \operatorname{HP}(E, \alpha)$ dan $x^{*} f \geq 0$ h.d pada E dan jika ACE sel maka

$$
x^{*}\left(x_{(f, A, \alpha)}\right) \geq 0
$$

Bukti : Karena $x^{*} f \geq 0$ h.d pada $E$ maka tidak mengurangi arti jika dianggap $x^{*} f(\bar{x}) \geq 0$ untuk setiap $\bar{x} \in E$ dan $x^{*} \in X^{*}$. Oleh karena itu, karena $\quad f \in \operatorname{HP}(E, \alpha)$, jadi untuk setiap $x^{*} \in X^{*}$ fungsi $x^{*} f$ terintegral Henstock pada $E$ dan untuk setiap sel $A \subset E$ terdapat vektor $x_{(f, A, \alpha)} \in X$ sehingga berakibat

$$
x^{*}\left(x_{(f, A, \alpha)}\right)=(\not) \int_{A} x^{*} f d \alpha \geq 0
$$
3.1.10

Di bawah ini merupakan akibat Teorema

Teorema 2.11. Jika $f \in \operatorname{HP}(E, \alpha)$ dan $g \in \operatorname{HP}(E, \alpha)$ dan $x^{*} f \leq x^{*} g$ h.d pada $E$ dan jika $A \subset E$ sel maka

$$
x^{*}\left(x_{(f, A, \alpha)}\right) \leq x^{*}\left(x_{(g, A, \alpha)}\right)
$$

Bukti : Karena $x^{*} f \leq x^{*} g$ h.d pada $E$ maka $x^{*}$ $g-x^{*} f \geq 0$ h.d. pada $E$. Oleh karena itu, berdasarkan Teorema 3.1.10 untuk setiap $x^{*} \in X^{*}$ fungsi $x^{*}(f-g)$ terintegral Henstock pada $E$ dan untuk setiap $A \subset E$ sel terdapat vektor

$$
\begin{aligned}
x_{(g-f, A, \alpha)} \in X \text { sehingga } \\
\begin{aligned}
x^{*}\left(x_{(g-f, A, \alpha)}\right) & =(\not{H}) \int_{A} x^{*}(g-f) d \alpha \geq 0 \\
& =(\not{H}) \int_{A} x^{*} g d \alpha-(\not{H}) \int_{A} x^{*} f d \alpha \geq 0 \\
& =x^{*}\left(x_{(g, A, \alpha)}\right)-x^{*}\left(x_{(f, A, \alpha)}\right) \geq 0
\end{aligned}
\end{aligned}
$$

Jadi

$$
x^{*}\left(x_{(f, A, \alpha)}\right) \leq x^{*}\left(x_{(g, A, \alpha)}\right)
$$

\section{DAFTAR PUSTAKA}

[1]. Dharmawidjaya, S., 2003, On The Bounded Interval Functions, Proceedings of the International Conference 2003 On Mathematics And Its Application, SEAMSGadjah Mada University, Universitas Gadjah Mada, Indonesia.

[2]. Indrati, Ch. R., 2002, Integral HenstockKurzweil pada ruang Euclide $\Re^{n}$ berdimensi- $n$, Disertasi, Universitas Gadjah Mada, Indonesia.

[3]. Gordon, R.A., 1994, The Integral of Lebesgue, Denjoy, Perron and Henstock, American Mathematical Society, USA.

[4]. Guoju, Ye and Tianqing, 2001.On HenstockDunford and Henstock-Pettis Integral, IJMMS, 25:7, Hindawi Publishing Corp. pp 467-478.

[5]. Guoju, Ye, and ,Swabik. S, 2001. The MacShane and The Weak. MacShane Intergal of Banach Space Value Function Defined On $\Re^{n}$. Mathematical Notes, Miscole, Vol, 2., No, 2., pp127-136.

[6]. Guoju, Ye, and Swabik. S, 2004, Topics in Banach Space Integration, manuscript in preparation.

[7]. Guoju, Ye, Lee, P.Y.,Wu, Congxin, 1999, Convergence Theorem of The DenjoyBochner, Denjoy-Pettis and Denjoy-Dunford Integral, southeast asian bulletin of mathematics, Springer-Verlag, vol 23; 135143.

[8]. Rahman, Hairur, 2005, Integral HenstockPettis pada ruang Euclide $\Re^{n}$, Tesis, Universitas Gadjah Mada, Indonesia. 
[9]. Rahman, Hairur, 2005, Kekonvergenan Integral Henstock-Pettis pada ruang Euclide $\mathfrak{R}^{n}$, Universitas Gadjah Mada, Indonesia.

[10]. Lee, P.Y., 1989, Lanzhou Lectures On Henstock Integration, Word Scientific, Singapore.

[11]. Lee, P.Y. dan Vborn, R., 2000, Integral: An Easy Approach after Kurzweil and Henstock, Cambridge University Press.
[12]. Pfeffer,W.F.,1993, The Riemann Approach to Integration, Cambridge University Press, New York, USA.

[13]. Royden, H.L., 1989, Real Analysis, third edition, Macmillan Publishing Company, New York, USA.

[14]. Swabik. S. and Ye, Guoju.,1991.The Macshne and The Pettis Intergal of Banach Space Value Function Defined on $\mathfrak{R}^{n}$, chzech, math journal. 